\title{
Reliability of Surface Electromagnetic Prospecting (SEP) system-A case study in Dongguashan copper mine
}

Da Lei, Xiaodong Luan,

Wenwei Zhang, Yangtao Ou

Key Laboratory of Shale Gas and Geoengineering, Institute of Geology and Geophysics

Chinese Academy of Sciences Beijing,China

\author{
Junjie $\mathrm{Wu}$, Xinchun Wang, \\ Yongbo Li \\ Institute of Geophysical and \\ Geochemical Exploration, \\ Langfang, China
}

\author{
Xiaodong Luan, Wen-Wei,Zhang \\ Yangtao $\mathrm{Ou}$ \\ University of Chinese Academy of \\ Sciences \\ Beijing, China
}

\begin{abstract}
The Surface Electromagnetic Prospecting system developed by Institute of Geology and Geophysics, Chinese Academy of Sciences was introduced through a CSAMT case study in Dongguashan copper mine in east of Tonglin, Anhui province. Strong electromagnetic noise generated by mineral industries and nearby downtown both challenges the electromagnetic field work and benefits the anti-interference test of the system. Measured data were however of good quality, which demonstrates that our system has the ability to stay reliable in complicate environment and can be employed in geophysical exploration practice.
\end{abstract}

Keywords-component; formatting; style; styling; insert (key words)

\section{INTRODUCTION}

The Development of Surface Electromagnetic Prospecting (SEP) system program leading by Institute of Geology and Geophysics, Chinese Academy of Sciences was started in 2010. It's one of the major research programs in the Sino-Probe Research Project supported by the Ministry of Land and Resources of China. The main objective of the program is to develop a Surface Electromagnetic Prospecting system with fully independent intellectual property, which can be used for MT, AMT, and CSAMT exploration. The goals have been achieved and many good results have been obtained ${ }^{[1]}$. Many field trial were conducted to test the new developed equipment in Gu'an, Zhangjiakou in Hebei province, Xin'cheng in Liaoning Province, and Jin'chang in Gansu Province, based on which, a lot of improvements have been made. During the field trial at Xin'he Neimenggu Province in 2013, a comparison experiment between our equipment and many leading foreign equipment include those from Zonge and Phoenix Company was made, and our system had showed very perfect behaviors $[2-3]$.

To investigate into the system's reliability in complicate environment furtherly, a cooperative exploration project was performed at Dongguashan copper deposit between Institute of Geology and Geophysics and Institute of Geochemistry, Chinese Academy of Sciences in 2014. Anti-interference experiment was carried out for v8 receiver from Phoenix Company, GDP32 receiver from Zonge Company, improved V8 ${ }^{[4]}$, and our equipment. Data of high quality can only be acquired by improved V8 and our equipment, which shows that the SEP system does have a strong anti-interference ability

\section{EM BACKGROUND OF DONGGUASHAN SURVEY}

Survey line BD-1 lay across the deposit, about $2.5 \mathrm{~km}$ in length. The site number are 77.5-125.5, with a offset of $50 \mathrm{~m}$ between every two nearest sites. The survey line was showed in Fig. $1^{[5]}$. Besides, some near surface electromagnetic noise sources are also demonstrated in Fig.1, which include power substations, signal tower, power lines, highways, railways, and pipe lines.

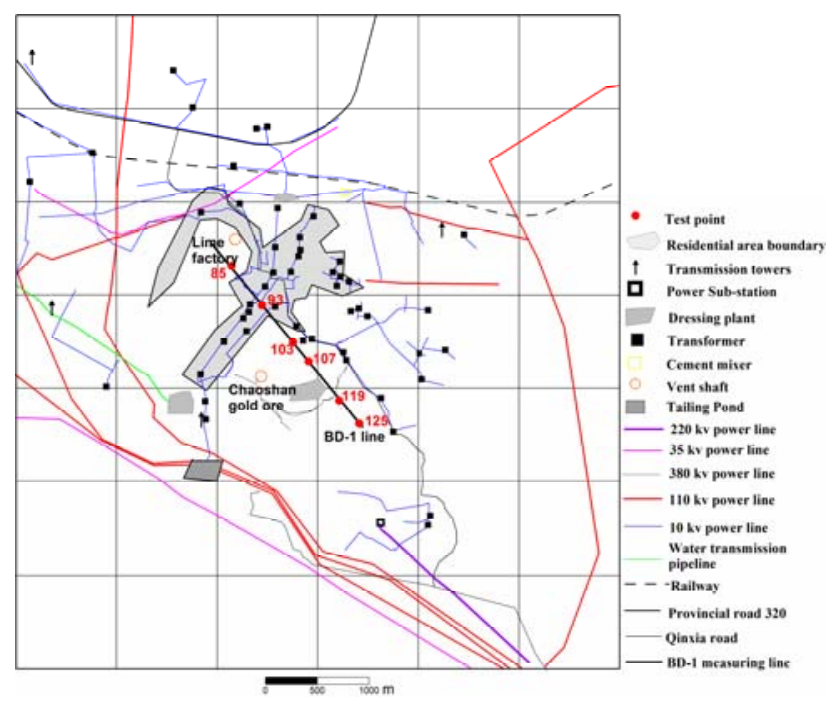

Fig. 1. Location of interference source in Dongguashan copper mine area

There are a power line of $220 \mathrm{kV}$, four power line of $110 \mathrm{kV}$, and another one of $35 \mathrm{kv}$ which circle our survey area and limit the survey line into a area of about $1-2 \mathrm{~km}$. what's more, the civil power lines of $220 \mathrm{~V}$ are distributed densely in that area. Three major power substations, including the Zhucun power substation, the largest power substation in East China are 
located there. Additionally, about 52 power voltage transformer of $10 \mathrm{kv}$ are distributed in the survey area, with 8 of which have a distance of less than $200 \mathrm{~m}$ to the nearest sites.

Qingxia Road, the main way from Zhucun town to Tonglin, was about $200 \mathrm{~m}$ to the north of survey line BD-1, which has a very heavy traffic vehicle. There is also a drilling gallery $200 \mathrm{~m}$ to the southwest of the BD-1 line with the depth of $800-1000 \mathrm{~m}$. Electronic facilities such as powerful donkey and winding engines and railways working in the gallery are operated $24 \mathrm{~h}$ a day and contribute to a very heavy electromagnetic noise to the survey.

\section{GEOLOGY OF DONGGUASHAN SURVEY}

Dongguasha copper deposit is a strata bounded skarn type copper deposit strictly controlled by the master strata. Metallic minerals contained are mainly pyrrhotite, pyrite, chalcopyrite and magnetite. The deposit has a very complex ore type, containing mainly copper pyrrhotite ore, followed by copper skarns, and copper pyrite ore. The main ore body lies in shaft portion and two wings of Qingshan anticline, strictly controlled by the Carboniferous, Huanglong to Chuanshan group. The top boundary of it usually lies in the contact zone between the uplifts portion of the anticline and the ore body, and up to the Permian Qixia group.

The Permian strata in our survey area show high resistivity and low polarization characteristics. The average resistivity is above $1000 \Omega \cdot \mathrm{m}$, and the average polarization rate is about 2$3 \%$. While the Permian Dalong group and Gufeng group have a resistivity as low as tens to hundreds $\Omega \cdot \mathrm{m}$, and polarization rate as large as $40 \%$, which are considered as interference to our survey. Intrusive rocks in this area all have moderate resistivity about hundreds $\Omega \cdot \mathrm{m}$, and polarization rate about $2-4 \%$. The electromagnetic properties of the deposit vary with the extent of mineration, mostly shows a low resistivity and high polarization trends. Its resistivity is usually less than $100 \Omega \cdot \mathrm{m}$, and polarization rate greater than $60 \%$.

\section{FIELD EXPERIMENTS}

\section{A. Equipment}

During the experiment of anti-interference, the CSAMT original data acquired employing V8 receiver were processed by two different programs. The current E2 version data preprocessing program developed by Phoenix Company takes only the $50 \mathrm{~Hz}$ noise into consideration, while neglects its high order harmonic waves. $\mathrm{Qi}$ and $\mathrm{Da}^{[6-7]}$ have discussed the employing of Notch filter to filtering the high order harmonic waves, and made improvement to the E2 version data preprocessing program. Their notch filter can suppress the 1-20th order harmonic waves successfully.

The powerful transmitter of SEP system has taken advantage of the transmitter has an output power of $50 \mathrm{kw}$, and could be extended to $100 \mathrm{kw}$. The maximum emissive current is up to 50A. The receiver of the system has a very broad frequency band from DC to $10 \mathrm{kHz}$. With a 24 bites A/D convertor, it has a large dynamic range bigger than $120 \mathrm{~dB}$. The suppression for power frequency noise is more than $70 \mathrm{~dB}$.
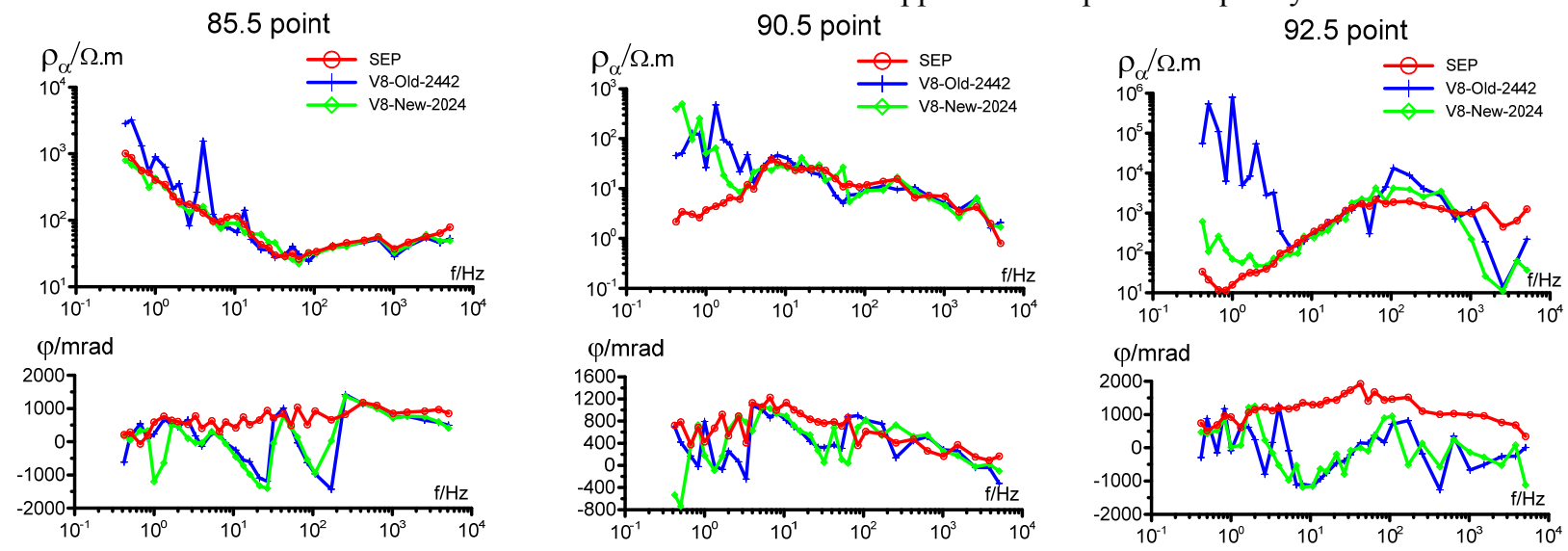

Fig. 2. the comparison between data acquired with V8 and SEP receivers

\section{B. Data Acquisition}

A broadside scalar observation configuration was employed for the CSAMT experiment. Ex and Hy components were recorded. Galvanic dipole source of $2300 \mathrm{~m}$ parallel to the BD1 line was injected into the earth. The offset was $8.1 \mathrm{~km}$.

Ground resistivity for receiver electrodes was less than $2000 \Omega$ through the usage of solid non polarizable electrodes. The length for electric field receiver dipole is $50 \mathrm{~m}$, with a 50 $\mathrm{m}$ distance every two dipoles. Electromagnetic signal of 40 frequencies from $0.42 \mathrm{~Hz}$ to $5120 \mathrm{~Hz}$ was emitted and received.

Two V8 receivers were employed for data acquisition, one with original software and one with modified software. They worked one after another at every site in line BD-1 sharing the same magnetic sensors and electrodes. After that, an array of six SEP receivers were put into practice.

\section{Results}

Survey line BD-1 run across some residential area with large population. A lot of power lines are distributed in that area. A lot of noise sources have already been shown in figure 2. Site 85.5 is located at a large quarry with a lot of facilities working day and night, which generated strong electromagnetic noise of a very broad band. As shown in Fig. 2, apparent resistivity and phase are serious biased by the noise at site 85.5 , 
especially for data acquired with V8 receiver. Data obtained with SEP system have a better performance. They are much smoother and have better resistance for noise. Sites 90.5 to sites 95.5 are located in the residential area and have a main drilling gallery beneath them. Strong electromagnetic background has a very bed influence on data acquired on those sites. Apparent resistivity and phase for Site 90.5 and 92.5 show that data acquired with V8 receiver are more likely to be biased by noise than SEP receiver.

\section{REFERENCE .}

[1] Teng J W. 2010. Strengthening exploration of metallic minerals in the second deep space on the crustal interior; Accelerated research development and industralization for Geophysical new technology and instrumental equipments. Progress in Geophys. (in Chinese), 25(3):729748.
[2] Di Q Y, Xu C, Fu C M, et al. 2015. Surface Electromagnetic Prospecting System(SEP) contrast testing Caosiyao molybdenum mine, inner mongolia. Chinese J. Geophys. (in Chinese),58(8):2065-2663.

[3] Di Q Y. 2015. Surface Electromagnetic Prospecting System(SEP) comparative test on Jichuan nickel mine of Gansu Province. Chinese J. Geophys. (in Chinese),58(10):3845-3854, doi:10.6038/cjg20151034.

[4] Qi Jianling, Lei Da,Wang Shumin, et al. 2009. A Study on InterferenceResistant Data Acquistion with Controlled Source Audio-Frequency Megnetotelluric Sounding Technique. International Symposium on Computer Science and Technology, ISCST, p589-593.

[5] Wu J J, Zhang J, Wang X C, et al. 2014. The analysis of electromagnetic noise characteristics in the Dongguashan ore district. Geophysical and Geochemical Exploration.(in Chinese),38(5):1-5.

[6] Lei D, Li X C, Zhao F G, et al. 2007. An application of spectrum analyzer to analyses of EM noises in mines. Computing techiques for Geophysical and Geochemical exploration. (in Chinese), 29(suppl): 0051 .

[7] Lei Da. 2010. Studies and applications of 2-D CSAMT modeling and inversion with a dipole source and topography. Chinese J. Geophys. (in Chinese), 53(4): 982-993. 